\title{
Effects of the Medium-Wall Modulus Contrast on the Pulse Wave Imaging (PWI) of Aortic Phantoms
}

\author{
Kexin Lin, Han Li and Danial Shahmirzadi \\ Department of Mechanical Engineering, Stevens Institute of Technology, Hoboken, NJ \\ dshahmir@stevens.edu
}

\begin{abstract}
Given its close correlation to cardiovascular pathologies, change in arterial stiffness has been deemed as a reliable biomarker for predicting cardiovascular events. The feasibility of using the ultrasound-based method of Pulse Wave Imaging (PWI) for estimating the arterial Pulse Wave Velocity (PWV) as surrogate of the wall stiffness has shown capacity for enhancing cardiovascular noninvasive diagnosis. The MoensKorteweg equation, which is the baseline for the modulus- $P W V$ relationship, does not account for factors such as the stiffness of the surroundings and the continuous pulsatile flow; while these factors are prevalent in phantom and in vitro PWI studies and could affects the PWV measurements. The objective of the present study is to quantify the correlation between the wave propagations and the stiffness of the surrounding medium, under various flow speeds. The $P W V$ results were quantified in terms of their $r^{2}$ and $S N R$, indicating the uniformity of the waves and variations in the measurements. The characteristic curves were developed in terms of the wall-to-medium modulus contrast that could be used as the reference for selecting the optimized gel and medium stiffness parameters that minimize the boundary conditions effects and maximize the $S N R$ and $r^{2}$.
\end{abstract}

Keywords- Pulse Wave Imaging (PWI); Aortic phantom in vitro; Modulus contrast; Pulse wave propagation

\section{Background}

Change in aortic stiffness has widely been reported as an independent indicator of all-cause and cardiovascular diseases (CVDs)-related mortalities such as primary coronary events, hypertension, aortic atherosclerosis and aneurysms [1-8], which can potentially leverage noninvasive estimation of arterial local stiffness and enhanced CVD diagnosis. Based on the formula known as the Moens-Korteweg (MK) equation, the velocity of the arterial pulse wave is correlated to the underlying wall stiffness $[9,10]$, and therefore can be used as a surrogate measure to estimate the arterial wall stiffness. Pulse Wave Imaging (PWI) is a recent, noninvasive, ultrasound-based technique to obtain a visual map of the arterial pulsatile wave propagations, and to estimate the regional Pulse Wave Velocity ( $P W V$ ) and wave propagation uniformity as denoted by the linear regression correlation coefficient, $r^{2}$ [11-13]. The feasibility of using PWI to effectively obtain a reliable estimate of $P W V$ has been shown in different applications such as simulation models [14-18], in vitro and in vivo animal models [19-21], clinical studies [22-25], and arterial phantom experiments $[13,21,26,27]$. Mimicking the arterial pulsatile flow conditions in a laboratory setup and performing PWI in vitro or on arterial phantoms have been found to be useful in optimizing the PWI technique, and to be helpful in gaining a better insight on the in vivo and clinical findings $[13,19,26,28,29]$.

DOI: $10.14738 /$ jbemi.34.2101

Publication Date: 22nd August 2016

URL: http://dx.doi.org/10.14738/jbemi. 34.2101 
Kexin Lin, Han Li and Danial Shahmirzadi; Effects of the Medium-Wall Modulus Contrast on the Pulse Wave Imaging (PWI) of Aortic Phantoms. Journal of Biomedical Engineering and Medical Imaging, Volume 3, No 4, August (2016), pp 18-29

In particular, tissue-mimicking arterial phantoms are effective and reliable substitutes for examining biological soft tissues of humans or animals [27]. The MK relationship for pulse wave velocity and wall stiffness has been theoretically developed for an impulse-generated wave that is traveling along an isolated tube with infinite length, constant circular ring cross section, and homogenous material, which is under a uniform external pressure $[9,10,30]$. However, the aforementioned underlying conditions do not exist in nearly any in vivo and in vitro applications of the Pulse Wave Imaging (PWI), and therefore the wave dynamics and the measured $P W V$ s could be altered from MK predictions. Particularly, in laboratory experimentations on arterial phantoms with finite length and constrained boundary conditions, the pulse wave propagation has been found to be a complex combination of various forward and reflected waves induced over multiple pulsatile cycles, which is also being affected by artifacts such as mechanical vibrations and rigid body motions of the phantom walls and the surrounding medium [13,26,29,31]. Furthermore, phantom walls are under constant incoming pulsatile flow, making wave dynamics further deviates from MK predictions, which is developed for free wave traveling initiated by a single impulse excitation of the walls. Understanding the wave dynamics in arterial phantoms under various tissuemimicking laboratory conditions helps interpreting the PWI findings in vitro, and implicating the findings to similar conditions in vivo. The experimental design parameters, most importantly the stiffness of the arterial phantom and the surrounding medium, have been found to play significant role in determining the dynamics of the wave propagation. The present parametric study is conducted to obtain qualitative and quantitative understandings of the effects of the wall and medium moduli on the pulse wave propagations and velocity, with the aim of providing characteristic curves to be used as a baseline for setting experimental design parameters.

\section{Materials and methods}

\subsection{Polyacrylamide phantom and Gel medium preparation}

Polyacrylamide (PA) phantoms have been shown to exhibit better stability over gel-based or agar-based phantoms [27,32] and thus were used to make the aortic phantoms in this study. In order to achieve phantoms with increasing stiffness, $20 \%, 30 \%$ and $40 \%$ concentrations of polyacrylamide were used, respectively, [13]; and agar was also added in order to induce speckles for enhanced imaging quality. Using a customized mold, phantoms with an inner and outer diameters of $d_{i}=6.41 \pm 0.79 \mathrm{~mm}$ and $d_{0}=9.89 \pm 0.61$ $\mathrm{mm}$, respectively, and the length of $\mathrm{L}=244.38 \pm 4.8 \mathrm{~mm}$, were made out of the resulted polyacrylamide mixtures. On the other hand, various gel media with increasing stiffness were obtained by mixing 60,150 , 300 and $400 \mathrm{~g}$ of gelatin (Now Foods: Beef gelatin natural powder) into $1 \mathrm{~L}$ of $90^{\circ} \mathrm{C}$ preheated water [33], respectively, resulting in 6, 15, 30 and $40 \% \mathrm{v} / \mathrm{V}$. In addition, water ( $0 \mathrm{~g}$ gelatin addition) was also used for the medium assimilating the lowest gel medium stiffness (i.e. $\approx 0 \%$ ). The gelatin medium, along with the PA phantom contained in, was left to cool down overnight, and was kept under $4^{\circ} \mathrm{C}$ refrigeration until shortly before the conduct of the new experiment. 


\subsection{Modulus characterization}

Compression mechanical testing was used to obtain the reference values for the modulus of polyacrylamide and gel materials. Biopsy specimens $(d=6 \mathrm{~mm})$ were prepared from all different concentrations of the polyacrylamide phantom samples as well as the gel medium samples. A rheometer (TA Instrument, DE, USA) was used to apply the compressive strain of $\varepsilon=5 \%$ at a strain rate of $\dot{\varepsilon}=0.5 \% / \mathrm{s}$. More details of the mechanical testing can be found in our reports elsewhere $[19,21]$. Alternatively, PWI method was used to estimate the modulus of the polyacrylamide phantoms based on the MK formula [910], as given below:

$$
P W V=\sqrt{\frac{E h}{\rho d\left(1-v^{2}\right)}} \quad \leftrightarrow \quad E=\frac{\rho d\left(1-v^{2}\right)}{h} P W V^{2},
$$

in which, PWV is the Pulse Wave Velocity, $E$ is the Young's modulus, $\rho$ is the density, $v$ is the Poisson's ratio, and $d$ and $h$ are the internal diameter and the wall thickness of the tube, respectively. The setup and protocol for conducting the PWI tests are described below.

\subsection{PWI experimental setup}

The PWI experiments in this study were performed on phantom/gel set up using combinations of the 3 different concentrations of PA phantoms and 5 different concentrations of gel media, leading to a total of 15 gel-to-phantom modulus contrasts, $E_{r}$, defined as $E_{r}=E_{G} / E_{P}$, where $E_{G}$ and $E_{P}$ are the gel and phantom Young's moduli, respectively. A customized laboratory setup was used to perform the PWI experiments. The setup consisted of a peristaltic pump (Manostat Varistaltic, IL, USA), providing a pulsatile flow into the phantoms in order to induce the pulsatile motion in the phantom walls, Fig. 1. Using a speed control knob, the flow rate (and velocity) can be controlled. The three fluid velocities of $0.106,0.113$ and 0.147 $\mathrm{m} / \mathrm{s}$ were examined in this study based on previous trials of measurement of $P W V$ on similar in-house aortic phantoms, and will be referred to as "Low", "Medium" and "High", respectively, throughout the rest of the manuscript. A Sonix Touch (Ultrasonix Medical, BC, Canada) ultrasound system with a $10 \mathrm{MHz}$, 16-beam, L14-5/38 linear array was used to acquire $38 \mathrm{~mm}$-wide B-mode images of the phantom walls at $950 \mathrm{fps}$ [25]. A radiofrequency (RF)-based, one-dimensional cross-correlation technique, described previously [34,35], was used to estimate the pulse-wave-induced displacements of the phantom wall, averaged over 10 cycles. The spatial and temporal information were transferred to generate a 2-D spatiotemporal map of the wall displacement, which was used to estimate the $P W V$ as the slope of the linear fit to the wave characteristic point, i.e. wave foot defined here as the $50 \%$ upstroke $[13,19,29,35]$. On each linear regression fit, the linear correlation coefficient $\left(r^{2}\right)$ was also obtained as a measure of the wave propagation uniformity. In order to comply with the MK assumptions, the most prominent forwardtraveling pulse wave (i.e. highest amplitude, isolated, homogeneously-propagating wave) in each of the 10 cycles was selected and used in PWV calculations. The variations in the PWV estimates were characterized by the average $(\mu)$ and standard deviation $(\sigma)$ of the measurements made over the 10 cycles. In order to assess the quality of the repeated data, the measure of the signal-to-noise $(S N R)$, defined as the ratio of the average of the PWVs to their standard deviation [36], is calculated as $S N R=\mu / \sigma$. 
Kexin Lin, Han Li and Danial Shahmirzadi; Effects of the Medium-Wall Modulus Contrast on the Pulse Wave Imaging (PWI) of Aortic Phantoms. Journal of Biomedical Engineering and Medical Imaging, Volume 3, No 4, August (2016), pp 18-29

\section{Results}

Figure 2 shows the mechanical testing characteristic curves of Young's modulus $(\mathrm{kPa})$ versus concentration (\%) for samples of both the polyacrylamide (PA) phantoms as well as the gel media. At each concentration, five specimens were tested $(n=5)$. The figure shows that both for the phantom and the gel, the modulus increases with increasing concentration, while the phantom always remains stiffer, i.e. modulus of 0-40 kPa, than the gel, i.e. modulus of 50-230 $\mathrm{kPa}$. Figure 3 illustrates the spatiotemporal maps of the wall displacement for four representative combinations of phantom and medium modulus contrasts, $E_{r}$. The figures illustrate the effects of wall-to-medium modulus contrast on the dynamics of the wave propagation, both qualitatively and quantitatively $-P W V$, linear correlation coefficient $\left(r^{2}\right)$, and signal-to-noise ratio $(S N R)$. The reflection waves have confounding effects on the characterization of the pulse wave velocities as related to the wall stiffness. In an in vitro aortic phantom set up like the one in the present study, the boundary conditions of fully-constrained ends of the phantom are responsible for generating nearly the entire reflective waves. Similar reflective waves and artifacts exist in vivo and have reported as potential sources of inaccuracies in $P W V$-based clinical methods such as the carotid-femoral pulse pressure measurements [13]. For instance, the asymmetric boundary conditions on the arteries caused by the stiff surrounding only on one side adjacent to the spinal cord, and the strong reflection at the site of the aortic bifurcations are among such conditions in vivo that makes the PWV-based conclusions deviating from the MK baseline. Figure 3 illustrates a change in the regimen of the wave propagation dynamics, as the mechanical properties of the wall and medium change, primarily shifting from a mechanical noise-driven vibrations for softer media toward increasingly-drained motions for the stiffer media, as high stiffness of the surrounding medium resists the free deflection of the tube walls. However, a balance could be obtained for a range of surrounding stiffness that makes the unnecessary mechanical vibrations fade away but does not kill the primary pulsatile waves. For instance in the Figs. 3.A-C, the linear fit to all the points on the primary wave yields very high correlation coefficients, i.e. $r^{2}>$ 0.86 , on the main forward waves. However, for very stiff medium (i.e. too much resistance against the wall motions), the magnitude of the main forward wave is decreased substantially, Fig. 3.D. Table 1 provides the average and standard deviation of PWV measurements, along with those of the $r^{2}$ for the measurements, over the entire 10 cycles, as laid out for all 15 combinations of wall and gel medium stiffness values. In order to examine the results in terms of the relative stiffness of the wall and medium, Fig. 4 displays the entire $P W V$ measurements from Table 1, as well as the associated $S N R$, in terms of the gel-to-phantom modulus contrast, $E_{r}=E_{G} / E_{p}$. A general trend of a gradual increase in the velocity of the wave travelling along the wall with increasing medium stiffness is observed; the velocity ranged from 0.72 $\mathrm{m} / \mathrm{s}$ in water (negligible stiffness) to $5.96 \mathrm{~m} / \mathrm{s}$ in $40 \%$ gel medium (highest stiffness). Having a continuous flow throughout the arterial tube is not an aspect that is considered in the MK equation, however, it is a factor that affects the wave dynamics. It was observed for example that for the $40 \%$ stiffness of the medium, the velocity increased from $3.77 \mathrm{~m} / \mathrm{s}$, to $4.52 \mathrm{~m} / \mathrm{s}$, and to $5.46 \mathrm{~m} / \mathrm{s}$, respectively, for low, medium and high, pump speeds. The PWVs ranged from $0.72 \mathrm{~m} / \mathrm{s}$ to $5.30 \mathrm{~m} / \mathrm{s}$ with the lowest and highest, respectively, occurring at phantom concentration of $20 \%$ with $0 \%$ gel concentration (i.e. water) in the pump low speed, and at phantom concentration of $40 \%$ with gel concentration of $40 \%$ (highest stiffness) at the pump high speed, respectively. Figure $4 . B$ elaborates on the signal-to-noise ratio (SNR) values corresponding to each $P W V$ measurement; the lower range of the spectrum, hovering just above $S N R \approx$ 
0 , is associated with the modulus contrast of $\approx 0$ (i.e. phantom concentration of $40 \%$ with gel concentration of $0 \%$ ) at pump low speed, while at the higher range of $S N R \approx 40$ lies the phantom concentration of $20 \%$ with gel concentration of $40 \%$ on the high speed setting, and the phantom concentration of $20 \%$ with gel concentration of $30 \%$ on the medium speed setting. Other combinations of gel and phantom modulus that result in pulse wave propagations with acceptable PWVs and SNRs can be concluded from Fig. 4.

\section{Discussion}

Noninvasive evaluation of Pulse Wave Velocity $(P W V)$ has widely been found effective in detection and examining the progression of various cardiovascular diseases [1-8]. Being a relatively recent technique, the feasibility of the ultrasound-based Pulse Wave Imaging (PWI) in producing a visual map of the estimated pulsatile wave with regional PWV estimates has been shown in different applications $[12,15,21,23-26,35,37-40]$. The $P W V$ estimate can be a surrogate for underlying tissue stiffness. Inferring conclusions on the wall stiffness based on the $P W V$ has primarily been derived from the formula known as MK, which is developed under several simplifying assumptions. Most notably, the MK predicts PWV of a wave which propagating along a cylindrical tube with constant cross-section, held in a constant pressure environment and being excited with a single impulse excitation. When examining a finite segment of the aorta in vivo, in vitro or on replica phantoms, embedded in a bath container, the dynamics of the aortic pulse wave and the $P W V$ deviate from Meons-Korteweg estimates, given that the underlying assumptions are not being met. In particular, existence of a stiff medium surrounding the aortic vessel as well as a continuous pulsatile flow through the vessel are not considered in the MK formulation, but have been found to be affecting the wave dynamics and the $P W V$ thereof $[13,26,29,31]$. However, it should be noted that the main objective of arterial PWI applications is not to estimate the absolute value of the wall modulus, but to identify the relative stiffening or softening of the wall; and such relative change in wall modulus have been shown to be captured effectively by the PWI method. The purpose of the present study was to quantify the understudied effects of the surrounding medium and the flow speed on the $P W V$ measurements to be able to determine the optimal design for a specific application. Intuitively, an extremely soft medium (i.e. low stiffness) would not contribute to elimination of the mechanical noise and undesired vibrations, while an extremely stiff medium (i.e. high stiffness) would fully constraint the phantom wall motions that not only kill the reflective waves, but also kill the main forward waves. Pulse wave propagations were examined on several combinations of wall and medium moduli, each under three different flow speeds, which are relevant to in vitro and in vivo applications. The pulse wave propagations were quantified based on the velocity of the pulsatile wave, $P W V$, the homogeneity of the wave propagation as characterized by $r^{2}$, and the consistency of the repeated measurements as characterized by SNR. Based on the findings, the best modulus combinations were noted to be the ones that had sufficient damping from the surrounding medium (namely the $30 \%$ and the $40 \%$ gel medium) along with the right combination of stiffness of the phantoms. In such cases, high-resolution and easy-to-deduce images were found that yield pronounced, highly correlated, main forward wave. For these particular combinations, the $P W V$ values were calculated with ease, owing to the high correlation coefficients of the forward waves, i.e. $r^{2}>0.86$. However, in the case of softer media (e.g. water), increased ratio of noise and the rigid body motions to the wall pulsatile motions contributed to higher variations in the $P W V$ 
Kexin Lin, Han Li and Danial Shahmirzadi; Effects of the Medium-Wall Modulus Contrast on the Pulse Wave Imaging (PWI) of Aortic Phantoms. Journal of Biomedical Engineering and Medical Imaging, Volume 3, No 4, August (2016), pp 18-29

measurements and SNR. Examining the effects of the flow speed, Table 1 shows that increasing pump speed may slightly decrease or increase the PWV for certain combination of phantom and gel moduli, plausibly due to the coupling of forced and natural motions of the wall at different resonance and intrinsic frequencies. Figure 4 provides the characteristic curves to be used as effective, versatile reference for gauging PWI experimental parameters. Twelve sets of $E_{r}$ shown on the abscissa and the $P W V$ and SNR measurements shown on separate plots enable determining the optimal set of medium stiffness and flow speed that yield consistent measurements of $P W V$, while maximizing the SNR.

\section{Conclusions}

The aim of this study was to quantify the effects of the phantom and gel medium moduli as well as the flow speed on the pulse wave propagation both qualitatively and quantitatively. Such effects have previously been found to play role in the wave dynamics, but were remained understudied. The findings provided characteristic curves that can be used to deduce the optimal modulus combination for a particular experimental design, in terms of the resultant pulse wave velocity $(P W V)$, linear correlation coefficient $\left(r^{2}\right)$, and signal-to-noise ratio $(S N R)$. For instance, a 20\% PA phantom in water was shown to yield a SNR close to zero with a poor, inconclusive linear fit, making up one end of the spectrum, while the $20 \%$ phantom in $40 \%$ gelatin resulted in an SNR of 39 forming the other end of the spectrum. It is concluded that the mere correlation between the wall modulus and $P W V^{2}$, as suggested by MoensKorteweg formula, is challenged for arterial PWI applications, and proper design and quantification of the parameters are needed. Future works need to be conducted on phantoms with more physiologicallyrelevant geometries, material properties and boundary conditions.

\section{ACKNOWLEDGEMENTS}

Danial Shahmirzadi would like to thank the director and members of the Ultrasound and Elasticity Imaging Lab (UEIL), Elisa Konofagou, Prathyush Narayanan and Ronny Li, respectively, at the Department of Biomedical Engineering of the Columbia University for assisting with the experimental set up and mechanical data, as well as for holding helpful discussions.

\section{REFERENCES}

[1] Kleinstreuer, C., Li, Z., and Farber, M. A., 2007, "Fluid-structure interaction analyses of stented abdominal aortic aneurysms," Annu Rev Biomed Eng, 9, pp. 169-204.

[2] Pereira, T., and Maldonado, J., 2010, "Comparative study of two generations of the Complior device for aortic pulse wave velocity measurements.," Blood Press. Monit., 15, pp. 316-321.

[3] Boutouyrie, P., Tropeano, A., Asmar, R., Gautier, I., Benetos, A., Lacolley, P., and Laurent, S., 2002, “Aortic stiffness is an independent predictor of primary coronary events in hypertensive patients: a longitudinal study," Hypertension, 39, pp. 10-15. 
[4] Laurent, S., Katsahian, S., Fassot, C., Tropeano, A. I., Gautier, I., Laloux, B., and Boutouyrie, P., 2003, “Aortic stiffness is an independent predictor of fatal stroke in essential hypertension," Stroke, 34, pp. 1203-1206.

[5] Sutton-Tyrrell, K., Najjar, S. S., Boudreau, R. M., Venkitachalam, L., Kupelian, V., Simonsick, E. M., Havlik, R., Lakatta, E. G., Spurgeon, H., Kritchevsky, S., Pahor, M., Bauer, D., and Newman, A., 2005, "Elevated aortic pulse wave velocity, a marker of arterial stiffness, predicts cardiovascular events in well-functioning older adults," Circulation, 111(25), pp. 3384-3390.

[6] Cruickshank, K., Riste, L., Anderson, S. G., Wright, J. S., Dunn, G., and Gosling, R. G., 2002, “Aortic pulsewave velocity and its relationship to mortality in diabetes and glucose intolerance: an integrated index of vascular function?," Circulation, 106, pp. 2085-2090.

[7] Woolam, G. L., Schnur, P. L., Vallbona, C., and Hoff, H. E., 1962, "The pulse wave velocity as an early indicator of atherosclerosis in diabetic subjects.," Circulation, 25, pp. 533-539.

[8] Blacher, J., Safar, M. E., Guerin, A. P., Pannier, B., Marchais, S. J., and London, G. M., 2003, "Aortic pulse wave velocity index and mortality in end-stage renal disease.," Kidney Int., 63, pp. 1852-1860.

[9] Moens, A., 1878, Die Pulskurve [The Pulse Curve], E.J. Brill., Leiden, The Netherlands.

[10] Korteweg, D. J., 1878, “Uber die Fortpflanzungsgeschwindigkeit des Schalles in Elastiischen Rohren," Ann Phys Chem, 5, pp. 52-537.

[11] Fujikura, K., Luo, J., Gamarnik, V., Pernot, M., Fukumoto, R., Tilson, M. D., and Konofagou, E. E., 2007, “A novel noninvasive technique for pulse-wave imaging and characterization of clinically-significant vascular mechanical properties in vivo," Ultrason Imaging, 29(3), pp. 137-154.

[12] Luo, J., Fujikura, K., Tyrie, L. S., Tilson, M. D., and Konofagou, E. E., 2009, "Pulse Wave Imaging of Normal and Aneurysmal Abdominal Aortas In Vivo," IEEE Trans. Med. Imaging, 28(4), pp. 477-486.

[13] Vappou, J., Luo, J., and Konofagou, E. E., 2010, "Regional measurement of arterial stiffness using Pulse Wave Imaging: Phantom validation and preliminary clinical results," Am J Hypertens, 23(4), pp. 393-398.

[14] Vappou, J., Zervantonakis, I., Luo, J., and Konofagou, E. E., 2008, "Finite Element Modeling of the Pulse Wave propagation in the aorta for simulation of the Pulse Wave Imaging (PWI) method," Comput. Biomech. Med. (MICCAI 2008 Work., pp. 118-127.

[15] Shahmirzadi, D., and Konofagou, E. E., 2012, "Detection of Aortic Wall Inclusions Using Regional Pulse Wave Propagation and Velocity In Silico," Artery Res., 6(3), pp. 114-123.

[16] Shahmirzadi, D., and Konofagou, E. E., 2014, "Quantification of arterial wall inhomogeneity size, distribution, and modulus contrast using FSI numerical pulse wave propagation," Artery Res., 8(2), pp. 5765. 
Kexin Lin, Han Li and Danial Shahmirzadi; Effects of the Medium-Wall Modulus Contrast on the Pulse Wave Imaging (PWI) of Aortic Phantoms. Journal of Biomedical Engineering and Medical Imaging, Volume 3, No 4, August (2016), pp 18-29

[17] Shahmirzadi, D., Jourard, I., and Konofagou, E. E., 2013, "Effects of the Sac Geometry and Modulus Contrast on the Regional Pulse Wave Propagation along the aneurysmal arterial wall in silico," International Tissue Elasticity Conference, Lingfield, UK, p. October 1-4.

[18] Shahmirzadi, D., and Konofagou, E. E., 2014, "Effects of Sac Geometry and Stiffness on Pulse Wave Propagations in FSI Models of the Human Abdominal Aortic Aneurysm," Cardiovasc. Eng. Technol., p. submitted.

[19] Shahmirzadi, D., Narayanan, P., Li, R. X., Qaqish, W. W., and Konofagou, E. E., 2013, "Mapping the longitudinal wall stiffness heterogeneities within intact canine aortas using Pulse Wave Imaging (PWI) ex vivo.," J. Biomech., 46(11), pp. 1866-1874.

[20] Luo, J., and Konofagou, E. E., 2011, "Imaging of Wall Motion Coupled With Blood Flow Velocity in the Heart and Vessels in Vivo: A Feasibility Study," Ultrasound Med. Biol., 37, pp. 980-995.

[21] Shahmirzadi, D., Li, R. X., and Konofagou, E. E., 2012, "Pulse-Wave Propagation in Straight-Geometry Vessels for Stiffness Estimation: Theory, Simulations, Phantoms and In Vitro Findings," J. Biomech. Eng., 134(11), p. 114502.

[22] Wang, S., Lee, W.-N., Provost, J., Luo, J., and Konofagou, E. E., 2008, “A composite high-frame-rate system for clinical cardiovascular imaging," IEEE Trans. Ultrason. Ferroelectr. Freq. Control, 55(10), pp. 2221-2233.

[23] Li, R. X., Luo, J., Balaram, S. K., Chaudhry, F. A., Shahmirzadi, D., and Konofagou, E. E., 2013, "Pulse wave imaging in normal, hypertensive and aneurysmal human aortas in vivo: a feasibility study," Phys. Med. Biol., 58(13), pp. 4549-4562.

[24] Li, R. X., Luo, J., Balaram, S. K., Chaudhry, F. A., Lantis, J. C., Shahmirzadi, D., and Konofagou, E. E., 2011, “Invivo Application of Pulse Wave Imaging for Arterial Stiffness Measurement under Normal and Pathological Conditions," 33rd Annu. Int. IEEE Eng. Med. Biol. Conf. Boston, MA, pp. 567-570.

[25] Luo, J., Li, R. X., and Konofagou, E. E., 2012, "Pulse wave imaging of the human carotid artery: an in vivo feasibility study," IEEE Trans. Ultrason. Ferroelectr. Freq. Control, 59(1), pp. 174-181.

[26] Li, R. X., Shahmirzadi, D., Qaqish, W. W., and Konofagou, E. E., 2012, "Performance assessment and optimization of Pulse Wave Imaging (PWI) and its applications for pulse wave analysis in ex vivo canine aortas and in vivo normal human arteries," 34th Annu. Int. IEEE Eng. Med. Biol. Conf., 2012, pp. 3179-82.

[27] Cloonan, A. J., Shahmirzadi, D., Doyle, B. J., Konofagou, E. E., and McGloughlin, T. M., 2014, "3D Printed Tissue-mimicking Phantoms for Medical Imaging and Computational Validation Applications," J. 3D Print. Addit. Manuf., p. submitted.

[28] Vappou, J., Luo, J., Okajima, K., Tullio, M. D., and Konofagou, E. E., 2011, "Aortic pulse wave velocity measured by pulse wave imaging (PWI): A comparison with applanation tonometry," Artery Res., 5(2), pp. $65-71$. 
Journal of Biomedical Engineering and Medical Imaging, Volume 3, No. 4, Aug 2016

[29] Shahmirzadi, D., Li, R. X., and Konofagou, E. E., 2012, "Pulse-Wave Propagation in Straight-Geometry Vessels for Stiffness Estimation: Theory, Simulations, Phantoms and In Vitro Findings," J. Biomech. Eng., 134(11), p. 114502.

[30] Fung, Y. C., 1997, Biomechanics: Circulation, Springer-Verlag, New York, NY.

[31] Zhang, X., and Greenleaf, J. F., 2006, "The stiffening of arteries by the tissue-mimicking gelatin.," IEEE Trans. Ultrason. Ferroelectr. Freq. Control, 53(8), pp. 1534-9.

[32] Ling, T., Jin, Q., Yao, H., and Zheng, H., 2010, “Design and Characterization of a Tissue-Mimicking Phantom for Ultrasonic Elastography," 2010 4th Int. Conf. Bioinforma. Biomed. Eng., pp. 1-4.

[33] Pavan, T. Z., Carneiro, A. a. O., Madsen, E. L., Frank, G. R., and Hall, T. J., 2009, “Exploring the nonlinear elastic behavior of phantoms materials for elastography," 2009 IEEE Int. Ultrason. Symp., (2), pp. 463-466.

[34] Luo, J., Lee, W.-N., and Konofagou, E. E., 2009, "Fundamental Performance Assessment of 2-D Myocardial Elastography in a Phased-Array Configuration," IEEE Trans. Ultrason. Ferroelectr. Freq. Control, 56(10), pp. 2320-2327.

[35] Luo, J., and Konofagou, E. E., 2010, "A fast normalized cross-correlation method for motion estimation," IEEE Trans Ultrason Ferroelectr Freq Control, 57(6), pp. 1347-1357.

[36] Bushberg, J. T., Seibert, J. A., Leidholdt, E. M., and Boone, J. M., 2001, The Essential Physics of Diagnostic Imaging, Lippincott Williams \& Wilkins, Philadelphia.

[37] Vappou, J., Luo, J., and Konofagou, E. E., 2010, “Pulse wave imaging for noninvasive and quantitative measurement of arterial stiffness in vivo.," Am. J. Hypertens., 23(4), pp. 393-8.

[38] Vappou, J., Luo, J., and Konofagou, E. E., 2010, "Pulse wave imaging for noninvasive and quantitative measurement of arterial stiffness in vivo," Am J Hypertens, 23(4), pp. 393-398.

[39] Vappou, J., Luo, J., Okajima, K., Tullio, M. D., and Konofagou, E. E., 2011, "Non-invasive measurement of local pulse pressure by pulse wave-based ultrasound manometry (PWUM)," Physiol. Meas., 32, pp. 16531662.

[40] Luo, J., Lee, W.-N., Wang, S., and Konofagou, E. E., 2008, “Pulse Wave Imaging of Human Abdominal Aortas In Vivo," Proc IEEE Ultrason. Symp, 2008, pp. 859-862. 


\section{LIST OF TABLES}

Table 1- PWV measurements (average \pm standard deviation) for different gel and PA phantom concentrations (and associated moduli), along with average and standard deviations for the $r^{2} s$ of the measurements. At each modulus combination, three increasing flow speeds of low $=0.106 \mathrm{~m} / \mathrm{s}$, medium $=0.113 \mathrm{~m} / \mathrm{s}$, and high $=0.147$ $\mathrm{m} / \mathrm{s}$ were examined.

\begin{tabular}{|c|c|c|c|c|c|c|}
\hline \multirow{3}{*}{\multicolumn{2}{|c|}{$\begin{array}{l}\quad \text { Gel Concentration } \\
\quad \text { (Modulus) } \rightarrow \\
\text { PA Concentration } \\
\text { (Modulus) } \downarrow\end{array}$}} & \multirow{3}{*}{$\begin{array}{c}0 \% \\
(0 \mathrm{kPa})\end{array}$} & \multirow{3}{*}{$\begin{array}{c}6 \% \\
(1.41 \mathrm{kPa}) \\
\end{array}$} & \multirow{3}{*}{$\begin{array}{c}15 \% \\
(8.21 \mathrm{kPa}) \\
\end{array}$} & \multirow{3}{*}{$\begin{array}{c}30 \% \\
(20.85 \mathrm{kPa})\end{array}$} & \multirow{3}{*}{$\begin{array}{c}40 \% \\
(36.96 \mathrm{kPa}) \\
\end{array}$} \\
\hline & & & & & & \\
\hline & & & & & & \\
\hline \multirow{3}{*}{$\begin{array}{c}20 \% \\
(51 \mathrm{kPa})\end{array}$} & Low speed & $\begin{array}{c}0.72 \pm 0.09 \\
\left(r^{2}=0.87 \pm 0.06\right)\end{array}$ & $\begin{array}{c}1.12 \pm 0.02 \\
\left(r^{2}=0.87 \pm 0.08\right)\end{array}$ & $\begin{array}{c}1.31 \pm 0.51 \\
\left(r^{2}=0.79 \pm 0.06\right)\end{array}$ & $\begin{array}{c}3.34 \pm 0.33 \\
\left(r^{2}=0.93 \pm 0.05\right)\end{array}$ & $\begin{array}{c}5.21 \pm 1.73 \\
\left(r^{2}=0.94 \pm 0.11\right)\end{array}$ \\
\hline & Med. speed & $\begin{array}{c}1.26 \pm 0.35 \\
\left(r^{2}=0.91 \pm 0.07\right)\end{array}$ & $\begin{array}{c}1.14 \pm 0.06 \\
\left(r^{2}=0.92 \pm 0.04\right)\end{array}$ & $\begin{array}{c}1.19 \pm 0.37 \\
\left(r^{2}=0.86 \pm 0.08\right)\end{array}$ & $\begin{array}{c}3.42 \pm 0.08 \\
\left(r^{2}=0.91 \pm 0.02\right) \\
\end{array}$ & $\begin{array}{c}5.96 \pm 1.03 \\
\left(r^{2}=0.92 \pm 0.04\right)\end{array}$ \\
\hline & High speed & $\begin{array}{c}1.73 \pm 0.42 \\
\left(r^{2}=0.89 \pm 0.03\right)\end{array}$ & $\begin{array}{c}3.11 \pm 0.49 \\
\left(r^{2}=0.89 \pm 0.04\right)\end{array}$ & $\begin{array}{c}0.79 \pm 0.47 \\
\left(r^{2}=0.89 \pm 0.03\right)\end{array}$ & $\begin{array}{c}3.06 \pm 0.34 \\
\left(r^{2}=0.96 \pm 0.03\right)\end{array}$ & $\begin{array}{c}3.13 \pm 0.08 \\
\left(r^{2}=0.90 \pm 0.07\right)\end{array}$ \\
\hline \multirow{3}{*}{$\begin{array}{c}30 \% \\
(110 \mathrm{kPa})\end{array}$} & Low speed & $\begin{array}{c}0.88 \pm 0.34 \\
\left(r^{2}=0.74 \pm 0.09\right)\end{array}$ & $\begin{array}{c}2.76 \pm 0.48 \\
\left(r^{2}=0.88 \pm 0.07\right)\end{array}$ & $\begin{array}{c}1.19 \pm 0.37 \\
\left(r^{2}=0.98 \pm 0.01\right)\end{array}$ & $\begin{array}{c}4.36 \pm 1.10 \\
\left(r^{2}=0.83 \pm 0.07\right)\end{array}$ & $\begin{array}{c}4.04 \pm 1.62 \\
\left(r^{2}=0.85 \pm 0.09\right)\end{array}$ \\
\hline & Med. speed & $\begin{array}{c}1.28 \pm 0.15 \\
\left(r^{2}=0.68 \pm 0.16\right)\end{array}$ & $\begin{array}{c}2.26 \pm 1.03 \\
\left(r^{2}=0.91 \pm 0.01\right)\end{array}$ & $\begin{array}{c}2.00 \pm 0.54 \\
\left(r^{2}=0.89 \pm 0.07\right)\end{array}$ & $\begin{array}{c}4.02 \pm 0.11 \\
\left(r^{2}=0.98 \pm 0.01\right)\end{array}$ & $\begin{array}{c}2.73 \pm 1.03 \\
\left(r^{2}=0.69 \pm 0.24\right)\end{array}$ \\
\hline & High speed & $\begin{array}{c}1.10 \pm 0.45 \\
\left(r^{2}=0.89 \pm 0.01\right)\end{array}$ & $\begin{array}{c}3.26 \pm 1.28 \\
\left(r^{2}=0.94 \pm 0.02\right)\end{array}$ & $\begin{array}{c}1.91 \pm 0.34 \\
\left(r^{2}=0.91 \pm 0.06\right)\end{array}$ & $\begin{array}{c}3.41 \pm 0.25 \\
\left(r^{2}=0.94 \pm 0.03\right)\end{array}$ & $\begin{array}{c}4.13 \pm 1.32 \\
\left(r^{2}=0.71 \pm 0.13\right)\end{array}$ \\
\hline \multirow{3}{*}{$\begin{array}{c}40 \% \\
(229 \mathrm{kPa})\end{array}$} & Low speed & $\begin{array}{c}1.91 \pm 1.27 \\
\left(r^{2}=0.82 \pm 0.08\right)\end{array}$ & $\begin{array}{c}3.77 \pm 1.29 \\
\left(r^{2}=0.90 \pm 0.08\right)\end{array}$ & $\begin{array}{c}2.29 \pm 0.55 \\
\left(r^{2}=0.96 \pm 0.03\right)\end{array}$ & $\begin{array}{c}5.06 \pm 0.28 \\
\left(r^{2}=0.98 \pm 0.01\right)\end{array}$ & $\begin{array}{c}2.78 \pm 0.20 \\
\left(r^{2}=0.76 \pm 0.12\right)\end{array}$ \\
\hline & Med. speed & $\begin{array}{c}1.14 \pm 0.46 \\
\left(r^{2}=0.78 \pm 0.12\right)\end{array}$ & $\begin{array}{c}4.52 \pm 0.30 \\
\left(r^{2}=0.86 \pm 0.09\right)\end{array}$ & $\begin{array}{c}1.79 \pm 0.41 \\
\left(r^{2}=0.89 \pm 0.07\right)\end{array}$ & $\begin{array}{c}4.73 \pm 1.26 \\
\left(r^{2}=0.93 \pm 0.03\right)\end{array}$ & $\begin{array}{c}3.00 \pm 0.45 \\
\left(r^{2}=0.84 \pm 0.04\right)\end{array}$ \\
\hline & High speed & $\begin{array}{c}2.65 \pm 0.37 \\
\left(r^{2}=0.87 \pm 0.04\right)\end{array}$ & $\begin{array}{c}5.26 \pm 0.45 \\
\left(r^{2}=0.91 \pm 0.07\right)\end{array}$ & $\begin{array}{c}2.29 \pm 0.67 \\
\left(r^{2}=0.91 \pm 0.06\right)\end{array}$ & $\begin{array}{c}5.25 \pm 0.68 \\
\left(r^{2}=0.87 \pm 0.11\right)\end{array}$ & $\begin{array}{c}5.30 \pm 2.02 \\
\left(r^{2}=0.75 \pm 0.13\right)\end{array}$ \\
\hline
\end{tabular}

\section{LIST OF FIGURES}

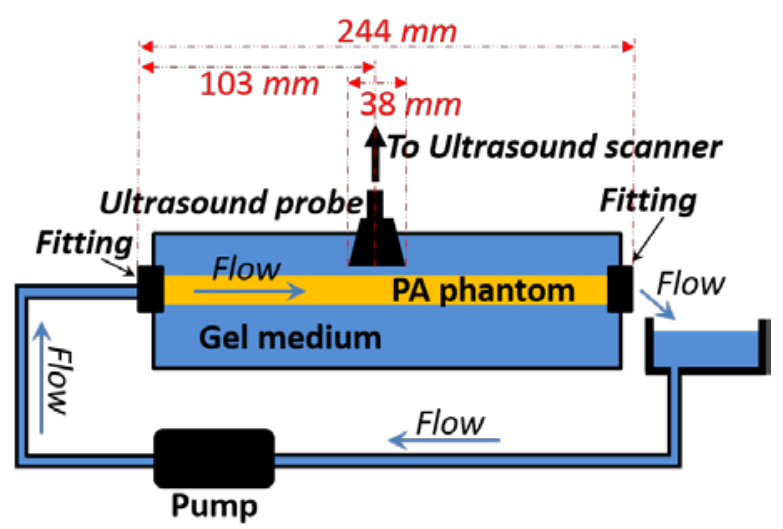

Figure 1- Schematics of the experimental set up for ultrasound Pulse Wave Imaging (PWI) of Polyacrylamide (PA) phantom, embedded in the gel medium. 


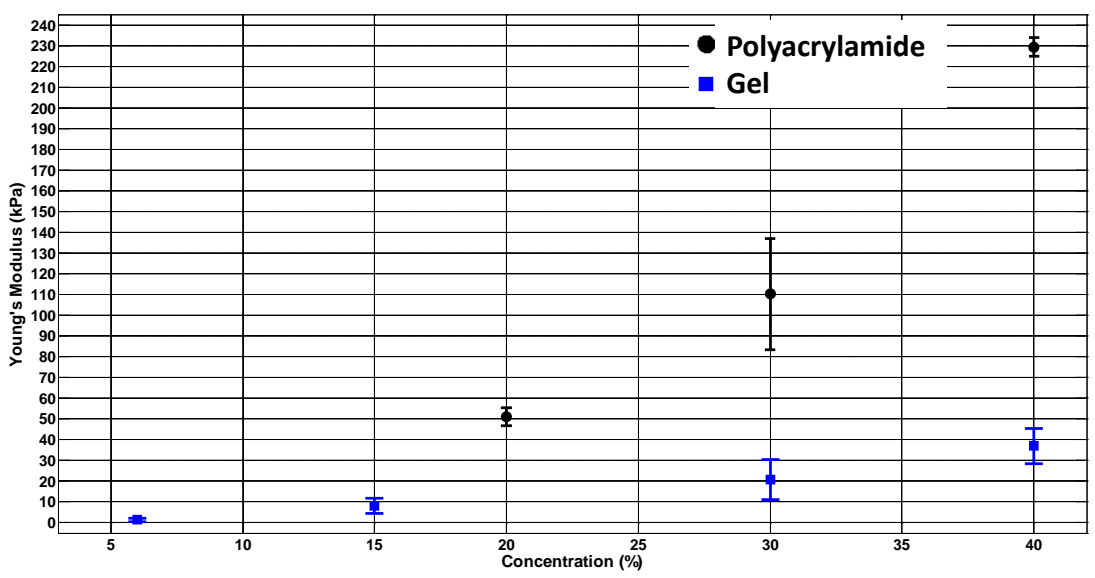

Figure 2- Young's modulus of Polyacrylamide (PA) phantom wall as well as gel medium, obtained under compressive mechanical testing.

(A) $30 \%$ GEL W. $20 \%$ PA (Er=0.409)

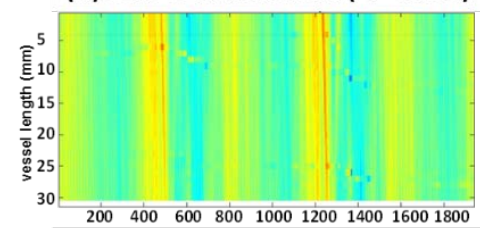

(B) $40 \%$ GEL w. $20 \%$ PA (Er=0.724)

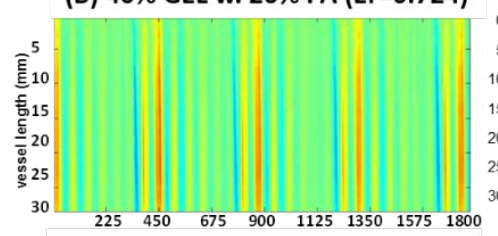

(C) $30 \%$ GEL w. $30 \%$ PA (Er=0.189)

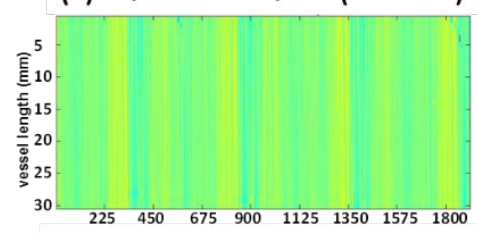

(D) $40 \%$ GEL w. $30 \%$ PA (Er=0.335)
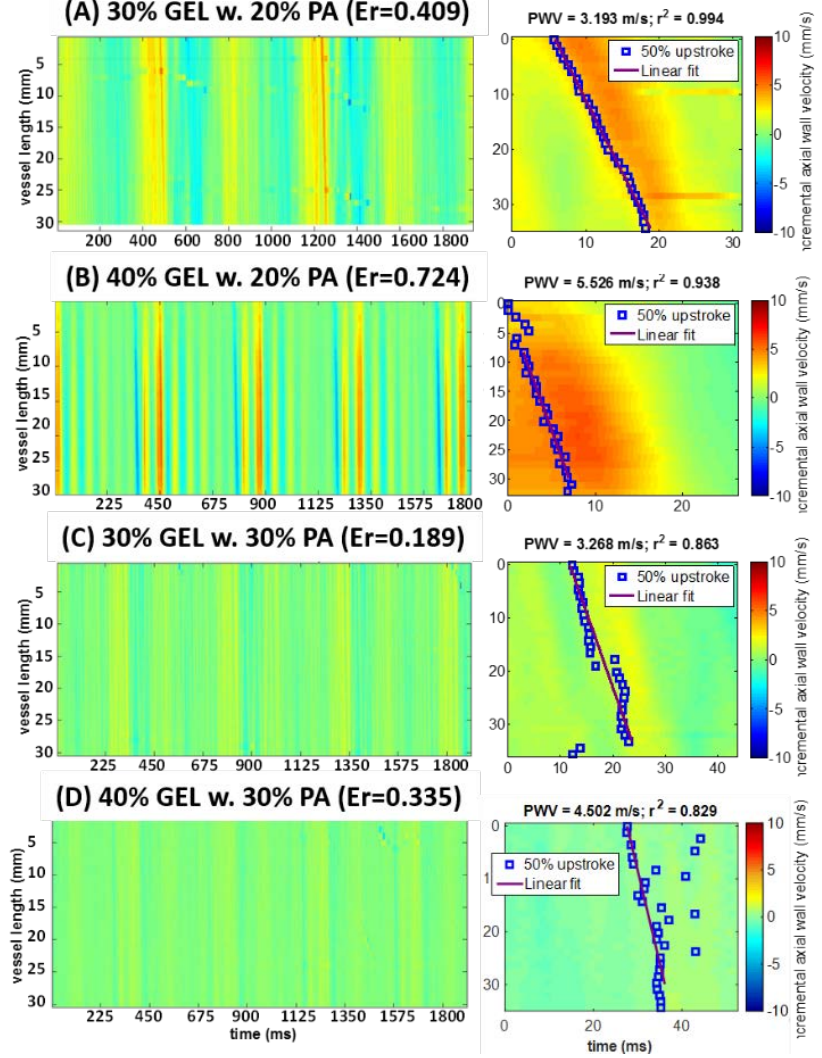

Figure 3- The spatio-temporal maps of the phantom wall displacements for representative gel-to-phantom modulus contrast ratios, $\mathrm{E}_{\mathrm{r}}$. The images on the left column show the wall displacements over multiple cycles, illustrating the overall wave propagations, while the images in the right column show the zoom-in on each map illustrating one dominant forward wave, used for PWV and $r^{2}$ measurements. 


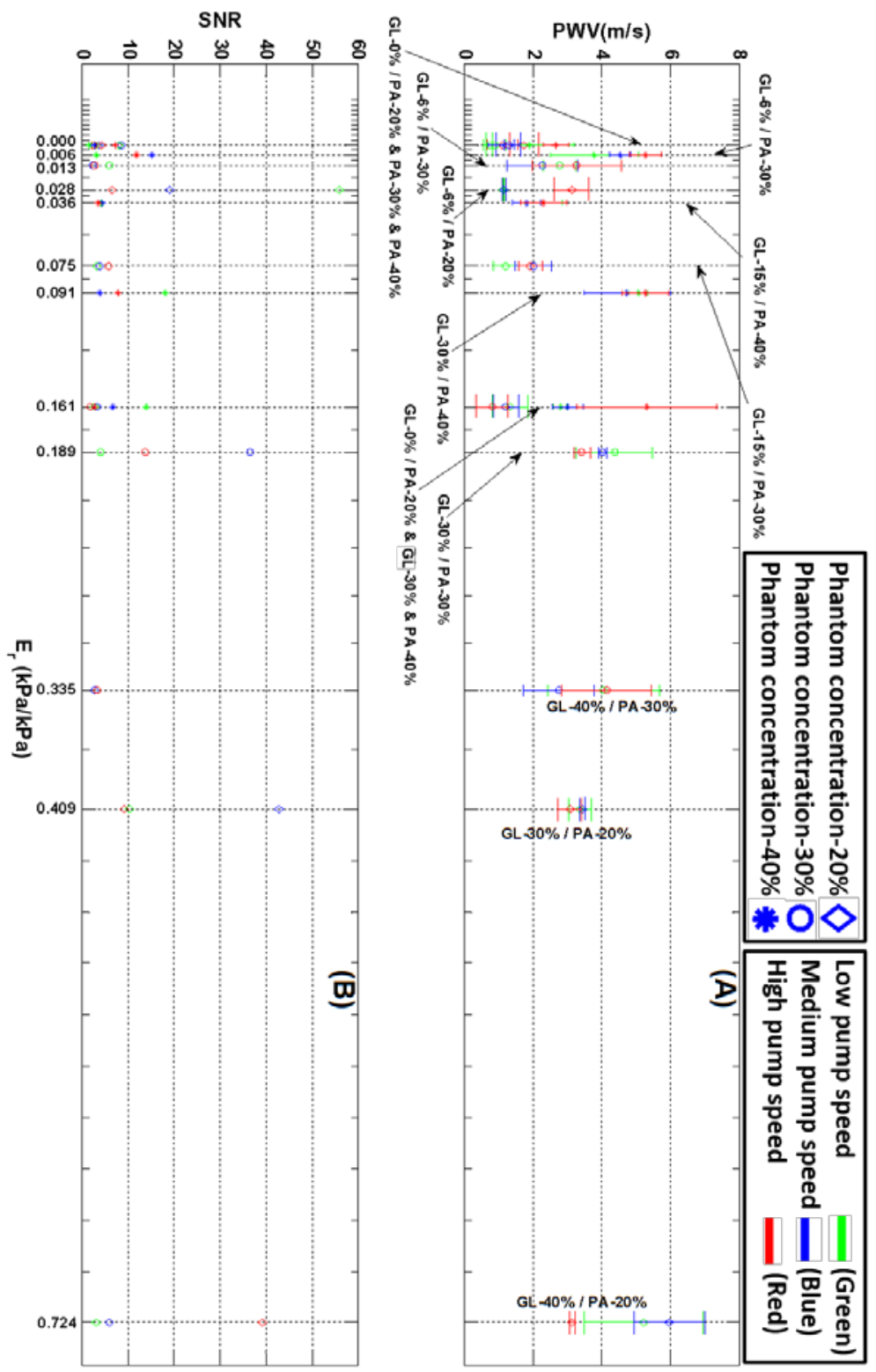

Figure 4- Characteristic curves of (A)Pulse wave velocity(PWV), and (B)Signal-to-noise ratio (SNR), for different gel-to-phantom modulus contrasts, $\mathrm{E}_{\mathrm{r}}$, and pump speeds. 